\title{
De l'ombre aux Lumières et réciproquement: redécouverte du crâne de Procavia capensis (Pallas, 1766) (Hyracoidea, Mammalia) décrit en 1767 par Daubenton dans l'Histoire naturelle
}

\author{
Rodolphe TABUCE \\ Institut des Sciences de l'Évolution (UM, CNRS, IRD, EPHE), \\ case courrier 064, université de Montpellier, Place Eugène-Bataillon, \\ F-34095 Montpellier cedex 05 (France) \\ rodolphe.tabuce@umontpellier.fr \\ (auteur correspondant)
}

Stéphane SCHMITT

CNRS, Laboratoire SPHERE (UMR 7219), Université Paris-Diderot CNRS, Bâtiment Condorcet, case 7093, 5 rue Thomas Mann, F-75205 Paris cedex 13 (France)

stephane_schmitt@yahoo.fr

Publié le 29 décembre 2017

urn:Isid:zoobank.org:pub:F8EBC844-C52B-475B-817A-CD663F912B3D

Tabuce R. \& Schmitt S. 2017. - De l'ombre aux Lumières et réciproquement: redécouverte du crâne de Procavia capensis (Pallas, 1766) (Hyracoidea, Mammalia) décrit en 1767 par Daubenton dans l'Histoire naturelle. Zoosystema 39 (4): 487-499. https://doi.org/10.5252/z2017n4a4

\section{RÉSUMÉ}

Un crâne de Procavia capensis (Pallas, 1766) (Hyracoidea, Mammalia), actuellement exposé dans la Galerie de Paléontologie et d'Anatomie comparée du Muséum national d'Histoire naturelle (Paris), est ici identifié comme celui que Daubenton décrivit en 1767, dans le cadre du catalogue des collections de quadrupèdes vivipares du Cabinet du Roi qu'il rédigea pour l'Histoire naturelle de Buffon. Tout en reconnaissant clairement dans ce crâne, originaire de Sidon (Liban), les caractères les plus remarquables des hyracoïdes, notamment la denture, Daubenton ne put alors identifier l'animal, dont la première description précise par Pallas parut pratiquement en même temps (fin 1766). Dans des volumes ultérieurs de l'Histoire naturelle, Buffon traita à plusieurs reprises du daman, sur la base de

MOTS CLÉS

Buffon,

Daman du Cap, Daubenton, histoire de l'anatomie comparée,
Hyracoides, données nouvelles, mais sans attribuer à cette espèce le crâne de Sidon, pourtant reconnu par Pallas dès 1776. Dans un volume de l'Histoire naturelle publié en 1789, après la mort de Buffon, ce crâne fut représenté sur une planche et considéré comme appartenant à un primate, le loris du Bengale (Nycticebus bengalensis (Lacépède, 1800)). Quinze ans plus tard, il fit partie des spécimens qui permirent à Cuvier de sortir le daman de l'ordre des Glires ou Rongeurs, dans lequel il était généralement placé depuis Pallas, pour le ranger dans celui des Pachydermes. 


\author{
KEY WORDS \\ Buffon, \\ Cape Hyrax, \\ Daubenton, \\ History of Comparative \\ Anatomy,
Hyracoids, \\ Pallas.
}

\begin{abstract}
From darkness to Enlightenment, and vice versa: rediscovery of the skull of Procavia capensis (Pallas, 1766) (Hyracoidea, Mammalia) described in 1767 by Daubenton in the Histoire naturelle.

A skull of Procavia capensis (Pallas, 1766) (Hyracoidea, Mammalia), currently on display in the Galerie de Paléontologie et d'Anatomie comparée (Paleontology and comparative Anatomy Gallery) of the Muséum national d'Histoire naturelle, Paris, is here identified as the one Daubenton described in 1767 in the catalog of the viviparous quadruped collections of the King's Cabinet he wrote for Buffon's Histoire naturelle (Natural History). While Daubenton clearly recognized in this skull from Sidon, Lebanon, the most remarkable characters of hyracoids, especially the teeth, he could not identify the animal, since the first precise description of the species was published by Pallas at the same time (at the end of 1766). In subsequent volumes of the Histoire naturelle, Buffon repeatedly dealt with the hyrax, on the basis of new data, but he failed to assign the skull of Sidon to this species, although Pallas recognized the nature of this skull as early as 1776. In a volume of the Histoire naturelle published in 1789, after Buffon's death, this skull was illustrated on a plate and considered as belonging to a primate, the Bengal slow loris (Nycticebus bengalensis (Lacépède, 1800)). Fifteen years later, it was one of the specimens which enabled Cuvier to remove the hyrax from the order Glires or Rodents, in which it was generally placed since Pallas, to assign it to the Pachydermes.
\end{abstract}

\section{INTRODUCTION}

L'ordre des hyracoïdes ne comprend aujourd'hui qu'une seule famille, les Procaviidae Thomas, 1892, qui regroupe cinq espèces au sein des genres Procavia Storr, 1780 (le daman des rochers), Heterohyrax Gray, 1868 (le daman des steppes) et Dendrohyrax Gray, 1868 (les damans des arbres) (Shoshani et al. 2013). D'un point de vue supra-ordinal, les hyracoïdes appartiennent aux paenongulés (avec les siréniens et proboscidiens) parmi les Afrotheria (Stanhope et al. 1998), terme qui fait évidemment référence à l'origine africaine supposée de ce clade de mammifères (Tabuce et al. 2008, Asher \& Seiffert 2010). Aujourd'hui encore, les hyracoïdes ont une répartition essentiellement africaine. Seule l'espèce Procavia capensis (Pallas, 1766) possède une aire de répartition qui s'étend hors d'Afrique; on la retrouve ainsi en Arabie, surtout à l'ouest et au sud, et au Moyen-Orient (Liban, Jordanie et Israël).

Les hyracoïdes sont longtemps restés inconnus des naturalistes européens. Les premières mentions et études, effectuées aux XVIIe et XVIIIe siècles, sont toutes relatives à l'espèce Procavia capensis, définie par Pallas (1766: 30). Les premiers représentants de cette espèce arrivent en Europe dans les ménageries et cabinets d'histoire naturelle au cours de la seconde moitié du XVIII siècle, d'abord en Hollande, puis à Paris. Les damans sont alors rangés dans l'ordre linnéen des Glires (voir par exemple Erxleben 1777: 352 et Gmelin in Linnaeus 1788: 166), puis, avec les réformes de la classification des mammiferes survenant à la fin du siècle, dans celui des rongeurs (Rosores chez Storr 1780, Rongeurs chez Geoffroy Saint-Hilaire \& Cuvier 1795). Cuvier (1804a et 1812: 4-12) est le premier à défendre l'idée qu'ils ne sont pas des rongeurs, mais des "pachydermes", et qu'ils se rapprochent donc des tapirs et des rhinocéros. Ses conclusions sont fondées sur l'étude de plusieurs spécimens venus de la région du Cap et d'un crâne provenant de la ville antique de Sidon, au Liban.
Avant d'être étudié par Cuvier, le crâne de Sidon avait été initialement décrit par Daubenton dès 1767 dans le catalogue des collections du Cabinet du Roi qu'il rédigeait alors pour l'Histoire naturelle, ouvrage qu'il avait entrepris depuis 1749 en collaboration avec Buffon (Buffon \& Daubenton 1767: 205-207). Cet article figurait dans le dernier volume consacré aux quadrupèdes, parmi plusieurs notices sur des objets arrivés récemment au Cabinet royal. Daubenton évoquait alors "la tête décharnée d'un animal inconnu aux Naturalistes» découverte dans un puits asséché de la ville antique de Sidon. En décrivant ainsi le crâne d'un animal non identifié, il entreprenait sans le savoir la première étude anatomique d'un crâne d'hyracoïde. C'est Pallas, en 1776, qui attribuera le premier ce crâne à Procavia capensis (alors nommé Cavia capensis). Curieusement, pourtant, il sera représenté plus tard dans les Suppléments de l'Histoire naturelle (Buffon 1789: 134-135 et pl. XXXVII : voir Fig. 1A) mais attribué alors à un primate, le «loris du Bengale».

Longtemps oublié, ce crâne a été redécouvert, deux cent cinquante ans après sa première description par Daubenton, dans les collections du Muséum national d'Histoire naturelle (héritier du Cabinet du Roi depuis la Révolution). Dans cet article, nous revenons sur la description du crâne proposée par Daubenton: en effet, celui-ci ne fait pas que décrire, il propose aussi quelques comparaisons intéressantes. Ainsi, les caractères crâniens et dentaires qu'il souligne sont bien ceux définissant aujourd'hui les hyracoïdes et témoignent de l'essor considérable de l'approche comparative en anatomie à cette époque, un essor dont il représente l'un des principaux acteurs. De manière significative, les conclusions auxquelles il parvient sont en totale concordance avec celles de Cuvier, près de quarante ans plus tard, à propos des damans. Afin de mieux saisir tout l'intérêt historique de ce crâne et avant d'analyser la pertinence de sa description par Daubenton, nous reviendrons dans un premier temps sur les connaissances relatives au groupe des hyracoïdes au XVIIIe siècle. 


\section{MATÉRIEL ET MÉTHODES}

Ce travail s'appuie sur la riche collection d'hyracoïdes actuels conservés au Muséum national d'Histoire naturelle (MNHN) de Paris (plus de 240 spécimens ostéologiques catalogués), sur les textes sources et leurs illustrations et enfin sur les catalogues du MNHN (e.g., Geoffroy Saint-Hilaire 1803; Fischer von Waldheim 1802-1803; Catalogue des préparations anatomiques du Cabinet d'Anatomie comparée du Muséum d'Histoire naturelle; Catalogue d'Anatomie comparée).

La redécouverte d'un objet décrit dans l'Histoire naturelle de Buffon et, plus généralement, de spécimens antérieurs à 1800, est assez exceptionnelle (pour d'autres exemples récents, voir Tassy 2002 à propos des ossements de mastodonte américain; Callou et al. 2010 à propos d'un tarsier naturalisé). En effet, il est rare que de tels spécimens, non seulement aient été conservés jusqu'à nos jours, mais qu'ils soient en outre reconnus comme tels, en raison tant des dégradations et des disparitions survenues au fil du temps que des pertes d'informations sur l'origine des objets lors des remaniements successifs des collections.

\section{RÉSULTATS ET DISCUSSION}

LES CONNAISSANCES SUR LES DAMANS AU SIÈCLE DES LUMIÈRES ET LEUR DESCRIPTION

DANS L'Histoire NATURELLE DE BufFon \& Daubenton $\mathrm{Au}$ XVIIe siècle et au début du XVIIIe, quelques rares auteurs (par exemple Hondius 1652: 24; Beaulieu 1664: 8; Kolb 1719: 159) mentionnent dans la région du Cap de BonneEspérance des «lapins», "blaireaux» ou "marmottes» que l'on peut attribuer (avec plus ou moins de certitude) à Procavia capensis. D'autre part, plusieurs observations effectuées depuis la Renaissance au Proche-Orient par des voyageurs, comme Alpini (dont le texte date du XVIe siècle mais n'est publié que beaucoup plus tard : voir Alpini 1735: 232) ou Shaw (1738: 376 et 1743: 75), se rapportent manifestement au daman. Alpini compare l'animal à un lapin et, pour le désigner, traduit en latin une dénomination locale, "agneau des fils d'Israël» (agnus filiorum Israel), tandis que Shaw retient le terme "daman", qu'il présente comme une transcription (manifestement fautive) du mot arabe désignant l'agneau, et emploie en anglais l'expression «Daman Israel». Cette dernière passe telle quelle dans la traduction française de sa relation, en 1743, et de là dans l'Histoire naturelle de Buffon, dont la grande diffusion va favoriser la stabilisation du terme "daman» en français, alors qu'il ne sera pas retenu en anglais. Ces premières deswcriptions, généralement sommaires, mettent l'accent sur les caractères extérieurs et le comportement (les «mœurs» et «habitudes») des animaux. Surtout, ces auteurs tendent, comme la plupart des Européens de cette époque, à rapporter tous les animaux inconnus qu'ils rencontrent à des espèces qu'ils connaissent déjà (lapins, marmottes...). En tout cas, aucun d'entre eux ne fait le rapprochement entre les formes vues en Afrique du Sud et celles du Proche-Orient.

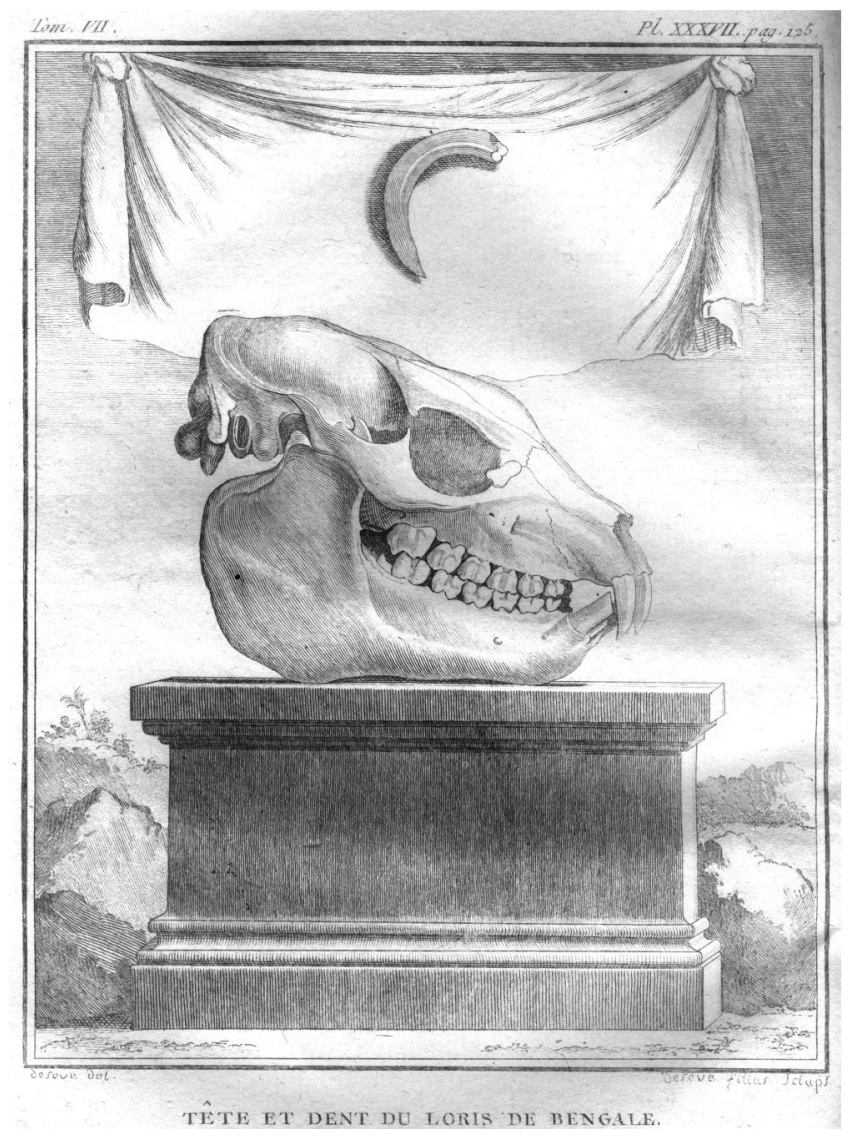

FIG. 1. - Reproduction de la planche XXXVII du Supplément de l'Histoire naturelle (tome 7 , publié en 1789) (Buffon 1789) figurant le crâne de Procavia capensis (Pallas, 1766) MNHN-ZM-AC-A.2339 alors attribué au loris du Bengale.

Les premiers damans font leur apparition en Europe dans des ménageries ou cabinets d'histoire naturelle aux Pays-Bas vers 1760, à la faveur des échanges avec la colonie néerlandaise du Cap. La plus ancienne mention certaine d'un spécimen ainsi importé est la description par Vosmaer, en 1767 , sous le nom de «marmotte bâtarde», de deux individus d'origine sud-africaine : l'un, jeune, conservé dans l'alcool et appartenant au cabinet du stathouder Guillaume V d'Orange, l'autre observé vivant dans une célèbre ménagerie d'Amsterdam, celle de Blaauw Jan (Vosmaer 1767a, b). Une fois mort, ce second animal est confié à Peter Simon Pallas (1741-1811), naturaliste allemand alors établi aux Pays-Bas (Wendland 1992: 48-57), afin d'être disséqué (Pallas 1766, 1767): il s'agit de la première étude anatomique d'un hyracoïde, qui est cependant incomplète car le crâne et les membres sont restés attachés à la peau, conservée pour la naturalisation de l'animal (Pallas 1766: 44). C'est à cette occasion que Pallas propose d'appeler l'animal Cavia capensis. Ainsi, contrairement à Vosmaer, plutôt que de le considérer comme une "marmotte», il préfère le placer dans le genre "Cavia» sensu Klein (1751: 49). Le genre Cavia comprend alors huit espèces dont "Cavia aguti» (aujourd'hui Dasyprocta leporina (Linnaeus, 1758), l'agouti), "Cavia cobaya» (aujourd'hui Cavia porcellus (Linnaeus, 1758), le cobaye) ou encore "Cavia paca" (aujourd'hui Cuniculus paca (Linnaeus, 1766), le paca); toutes ces espèces sont américaines 
et aujourd'hui classées parmi les Cavioidea, une super-famille de rongeurs hystricognathes (voir Patton et al. 2015). Pour Pallas, l'attribution de l'espèce capensis au genre Cavia se fonde en particulier sur la morphologie des membres et la formule dentaire, qui sont au XVIII siècle parmi les principaux critères de classification des mammifères. Il relève notamment un membre antérieur tétradactyle et la présence de deux incisives supérieures suivies d'un long espace (diastème), d'une petite dent accessoire et de quatre "molaires» de grande taille. Ces quatre dents postérieures, par leur nombre et leur taille, ne sont pas sans rappeler effectivement celles des Cavioidea. Cette similitude a toutefois ses limites. En effet, s'il est aujourd'hui bien établi que la formule dentaire des cavioïdes comporte une seule prémolaire de grande taille (la quatrième), suivie de trois molaires de taille équivalente, il s'avère que celle des hyracoïdes est constituée de quatre prémolaires et trois molaires de taille croissante. La position de Pallas s'explique toutefois par une raison simple : il a disséqué un jeune individu ayant encore sa denture lactéale, à savoir une canine (la petite dent accessoire citée par Pallas, ce locus dentaire n'est pas remplacé chez l'adulte) et quatre prémolaires déciduales molariformes. Ajoutons enfin que, si Pallas rapporte au genre Cavia l'espèce C. capensis, il fait remarquer qu'elle s'y distingue par des différences anatomiques importantes : il reconnaît qu'il s'agit d'une "espèce anomale» dans le genre et que «la conformation de cet animal est à plusieurs égards insolite et remarquable» (1767: 18-20).

Ce sont ces travaux, en particulier celui de Vosmaer, que Buffon va longuement reprendre dans son article de 1776 "De la Marmotte du Cap de Bonne-Espérance» (Buffon 1776: 177-180 et pl. XXIX). D’ailleurs, conformément à une pratique assez fréquente dans les volumes du Supplément de l'Histoire naturelle, la gravure qu'il publie est une copie quelque peu modifiée de celle de Vosmaer (dont Pallas s'était déjà largement inspiré également). La seule contribution significative de Buffon est alors son argumentaire qui lui fait préférer le terme de "marmotte » à celui de «cavia» : en effet, ce dernier, selon lui, est particulièrement impropre car il est d'origine brésilienne et doit donc désigner uniquement des espèces américaines. Cette distinction entre les espèces de l'Ancien et du Nouveau Monde, surtout celles des régions méridionales qui n'ont pu a priori migrer d'un continent à l'autre, est un point extrêmement important dans la théorie des climats de Buffon, qui préfère se fier à des critères biogéographiques plutôt que morphologiques pour distinguer et nommer les espèces (voir notamment Buffon 2016: 345-370 et Schmitt, à paraître).

Par ailleurs, dans ce même chapitre, Buffon cite une lettre et une gravure envoyées par Allamand à Daubenton (courrier qui, d'après le texte, pourrait être antérieur aux travaux de Vosmaer et Pallas) à propos d'un autre spécimen naturalisé du musée de l'Académie de Leyde. Allamand y utilise, mais non sans exprimer des doutes à ce sujet, le mot «cabiai», ce qui pourrait indiquer qu'il a eu lui-même l'idée de placer cet animal dans le genre Cavia, à moins que ce ne soit Pallas qui le lui ait suggéré. Indépendamment de ce passage de 1776 concernant des damans venus d'Afrique du Sud, Buffon avait évoqué dès 1765 le daman du Proche-Orient en se référant alors uniquement à la littérature, plus précisément à Shaw et Alpini : il le rangeait alors parmi les «gerboises" (Buffon \& Daubenton 1765: 143, 148-149). En 1776, il ne fait pas du tout le rapprochement entre les deux animaux.

Mais, en 1782, dans un nouveau volume du Supplément à l'Histoire naturelle, il consacre un chapitre particulier au «daman-Israël» (1782: 276-282 et pls XLII et XLIII) (Fig. 2). Il revient alors sur les observations et conclusions d'Alpini et de Shaw relatives à la forme proche-orientale, mais cette fois sur la base d'informations inédites que lui a transmises (avec une illustration) le voyageur James Bruce, parti en Afrique à la recherche des sources du Nil (sa relation sera publiée plus tard; sur le daman, voir Bruce 1790: 241). Or, pour la première fois, Buffon se rend compte des similitudes de ce «daman-Israël», non seulement avec un animal du Cap envoyé récemment par Sonnerat (spécimen possiblement collecté en 1781; Rookmaaker 1989: 38), mais aussi avec celui qu'il a appelé en 1776 «marmotte du Cap » et qu'ont décrit Pallas et Vosmaer. Il ajoute en outre des données inédites sur cette forme sud-africaine, publiées entre-temps dans l'édition hollandaise de l'Histoire naturelle par Allamand, qui a employé quant à lui le nom néerlandais de "klip-das", c'est-à-dire littéralement "blaireau des rochers» (Buffon 1778: 157-160). Certes, Buffon distingue les deux espèces, sous les noms respectifs de «daman-Israël» (Fig. 2) et de "daman du Cap», mais il admet qu'il existe entre elles « la plus grande ressemblance par la grandeur \& la conformation, par le nombre des doigts $\&$ par le manque de queue».

Cette même année, Daubenton, qui entreprend la partie zoologique de l'Encyclopédie méthodique, reconnaît lui aussi les deux espèces de daman décrites et nommées par Buffon, dont il se contente d'ailleurs de citer des extraits (Daubenton 1782: 102-103). D'autre part, toujours en 1782, un aristocrate et naturaliste amateur allemand, Mellin, publie une petite monographie consacrée au daman du Cap, pour lequel il préfère, comme Allamand, employer la dénomination de «Klipdas». Cuvier (1822: 128, note de bas de page) considèrera ce travail comme «la meilleure description et histoire du daman ".

Une contribution de Mellin apparaît aussi dans l'Histoire naturelle. En effet, dans le septième et dernier volume du Supplément, paru un an après la mort de Buffon par les soins de Lacépède, se trouve un ultime article sur le daman, intitulé "Addition et corrections à l'article de la Marmotte du Cap de Bonne-Espérance" (Buffon 1789, 7: 308-312 et pl. LXXIX). Ce texte discute en particulier l'usage des termes «marmotte» et «blaireau », qu'il rejette au profit de celui de klipdas. À l'appui de cette décision, est reproduite une longue lettre de Mellin, ainsi que la gravure qui l'accompagnait; en revanche, l'article publié par Mellin (1782) n'est pas cité. En outre, toute la discussion de 1782, qui identifiait la «marmotte du Cap» avec le «daman-Israël», est totalement ignorée, ce qui suggère que ce texte paru en 1789 a été rédigé beaucoup plus tôt, sans doute avant 1782 , et a été publié sans grand discernement par Lacépède qui a dû le trouver tel quel dans les papiers laissés par Buffon. 
On voit donc qu'à la fin du XVIII e siècle, essentiellement grâce aux travaux de Pallas, d'Allamand et de Buffon, deux résultats importants ont été acquis relativement aux damans : en premier lieu, le rapprochement du daman décrit par les voyageurs au Proche-Orient avec un animal d'Afrique du Sud jusqu'alors diversement nommé («marmotte», etc.); en second lieu, la reconnaissance du caractère assez inhabituel de leur morphologie, notamment dentaire. Ces travaux reposent, d'une part, sur l'observation d'exemplaires venus de la colonie néerlandaise du Cap, d'autre part, concernant les formes proche-orientales, sur la littérature (Shaw, Alpini). En revanche, avant 1800, jamais le crâne libanais décrit par Daubenton en 1767 et illustré en 1789 (Fig. 1A; voir infra, troisième partie) n'est rapproché du "daman", de la "marmotte du Cap» ou du «klipdas» par les auteurs de l'Histoire naturelle. Seul Pallas (1776: 85-86) comprend qu'il s'agit du même animal, mais sa remarque à ce sujet ne sera pas exploitée avant 1804 .

\section{L'ESSOR DE L'ANATOMIE COMPARÉE ET LES VARIATIONS DE LA POSITION DES DAMANS (1766-1804) : \\ DE RONGEURS ATYPIQUES À PACHYDERMES}

Pallas range donc le daman, qu'il nomme Cavia capensis, parmi les Glires (littéralement, "loirs»), un ordre créé par Linnaeus dès 1735 et comprenant surtout des rongeurs et des lagomorphes au sens actuel, mais aussi, au gré des éditions du Systema Nature, le rhinocéros (Rhinoceros Linnaeus, 1758, éditions X et XI), des chiroptères (Noctilio Linnaeus, 1766, édition XII), des musaraignes (Sorex sensu Linnaeus, 1735, éditions I-III et VI-IX) et des marsupiaux (Didelphis sensu Linnaeus, 1748, éditions VI-IX). Cette position systématique du daman est conservée par divers auteurs, notamment Erxleben (1777: 352) et Gmelin dans sa réédition du Systema Nature de Linnaeus (1788: 166). En revanche Storr, qui remanie sensiblement la classification des mammifères en 1780, remplace l'ordre des Glires par celui des Rosores («rongeurs») et retire le daman du genre Cavia, au motif que c'est un animal africain et non américain, et lui consacre un genre particulier, Procavia Storr (1780: 40). Hermann (1783: 115), quant à lui, conserve l'ordre linnéen des Glires mais donne au daman un nouveau nom, Hyrax capensis (du grec hurax, désignant chez Nicandre un petit animal non identifié, rongeur ou insectivore). Ce nom est retenu, entre autres, par Gmelin et par Geoffroy Saint-Hilaire \& Cuvier (1795: 173, 180) : mais tandis que Gmelin garde encore les Glires de Linnaeus, Geoffroy et Cuvier situent le daman ou Hyrax dans l'ordre des Rongeurs qui correspond à peu près aux Rosores de Storr.

Ainsi, avant 1800 , quel que soit le nom du genre dans lequel il est placé, la position du daman dans l'ordre des Glires ou dans celui des Rongeurs (ou Rosores), qui en est grosso modo l'héritier, n'est généralement pas contestée par les naturalistes, lesquels se fondent alors principalement, comme Pallas, sur le critère traditionnellement employé pour la classification des mammiferes, à savoir la denture (ici la présence d'une grosse paire d'incisives supérieures suivies d'un diastème). Curieusement, le seul auteur à faire exception, Vicq d'Azyr, place le daman (ou «marmore», comme il appelle l'animal, soit volontairement,

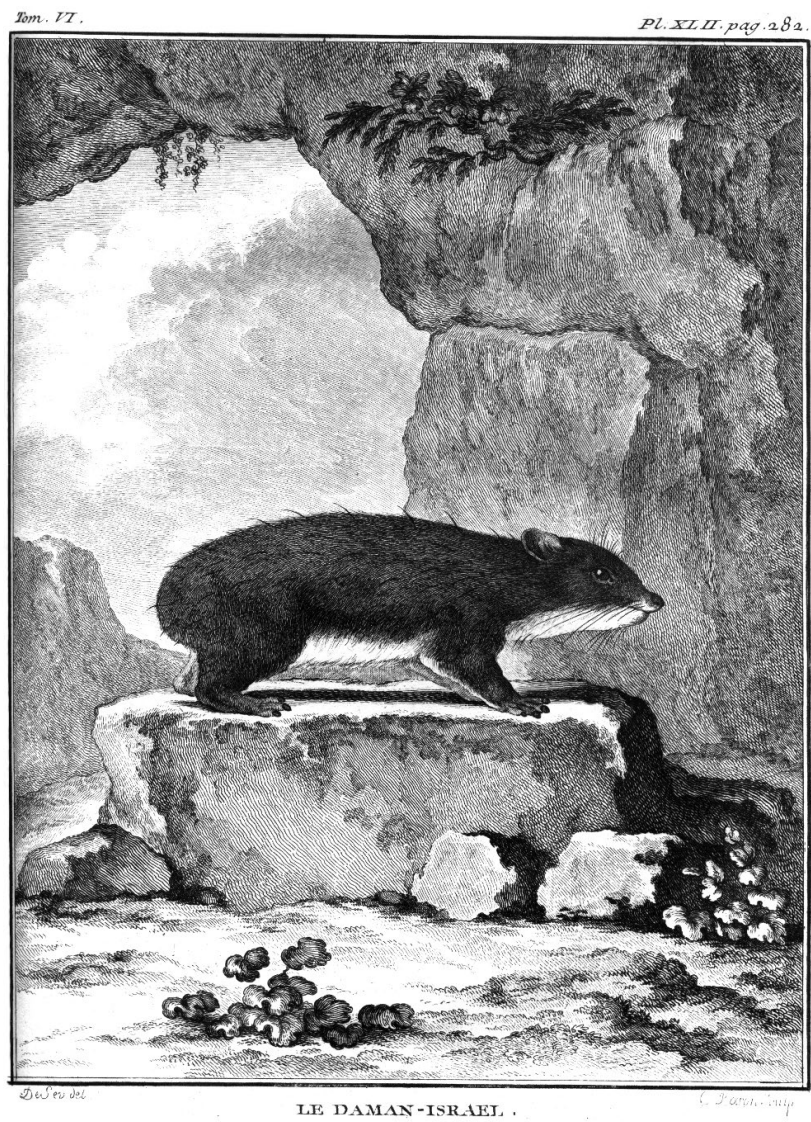

FIG. 2. - Reproduction de la planche XLII du Supplément de l'Histoire naturelle (tome 6, publié en 1782) (Buffon 1782) illustrant le "daman-Israël » (= Procavia capensis (Pallas, 1766)).

soit à la suite d'une coquille; Vicq d'Azyr 1792: ciii, 359) avec les musaraignes dans sa "classe» des Soriciens, la cinquième de l' "ordre» des quadrupèdes vivipares (il inverse en effet ces catégories taxonomiques), mais il s'appuie également sur la denture pour justifier ce rapprochement (1792: ciii). Selon lui, les Soriciens possèdent "deux dents incisives alongées (sic) à la mâchoire supérieure; les incisives, les canines $\&$ les molaires contiguës »; sur la base de cette définition on peut donc s'étonner de cette position du daman dans la classification proposée par Vicq d'Azyr (en effet les damans n'ont pas de canine chez l'adulte et possèdent un diastème entre les incisives et la rangée molaire, voir plus haut).

Dans les dernières décennies du XVIII siècle, l'idée se répand parmi les naturalistes que la classification des animaux doit reposer sur l'étude de leur organisation interne plutôt que sur des caractères externes. L'anatomie comparée connaît alors un essor considérable, notamment sous l'impulsion de Daubenton et de Vicq d'Azyr (Farber 1975; Balan 1979; Schmitt 2006, 2009, 2016). Ce dernier, qui meurt en 1794, n'a guère le temps de mettre en application son programme d'une systématique reposant sur l'anatomie, et, nous l'avons vu, sa classification de 1792 est encore largement fondée sur la morphologie externe, de même que celle proposée par Cuvier et Geoffroy Saint-Hilaire en 1795. Mais dans les années qui suivent, émergent de nouvelles tentatives. 
Ainsi, dès 1800, les Leçons d'anatomie comparée de Cuvier établissent les fondements de cette nouvelle approche, et déjà il affirme que «le daman (hyrax) [...] doit être rapporté à l'ordre des pachydermes et non à celui des rongeurs ", sans toutefois donner d'argumentaire plus précis (Cuvier 1800: 66). À l'inverse, Wiedemann (1802), décrivant de manière détaillée le crâne du daman, parvient également à la conclusion qu'il ne peut être rangé parmi les rongeurs, mais sans pour autant proposer de classification alternative.

C'est surtout dans un article de 1804 (reproduit dans 1812, 2: 4-12) que Cuvier développe ses vues sur le daman, une espèce qui illustre à elle seule, selon lui, l'intérêt de l'approche anatomique : "Il n'est point de quadrupède, dit-il, qui prouve mieux que le daman la nécessité de recourir à l'anatomie pour déterminer les véritables rapports des animaux" (Cuvier 1804a: 171). Pour réaliser son étude, Cuvier dispose alors de plusieurs exemplaires de damans. Les données qu'il mentionne et celles qui sont disponibles dans certains catalogues du MNHN (Geoffroy Saint-Hilaire 1803: 237-238; Fischer von Waldheim 1803: 127, 329 et 343; Catalogue des préparations anatomiques du Cabinet d'Anatomie comparée du Muséum d'Histoire naturelle) suggèrent au moins une dépouille naturalisée, un spécimen très jeune conservé dans l'alcool et son probable squelette (celui-ci est figuré; Cuvier 1804a: pl. 18), au moins un squelette entier (dont les tarse et carpe sont figurés; Cuvier 1804a: pl. 18) et au moins deux crânes illustrant un individu adulte du Cap et un individu sub-adulte du Liban. Ce dernier spécimen est celui qu'avait décrit Daubenton en 1767 (voir à ce propos Cuvier 1822: 134, note de bas de page). Sa vue latérale, donnée par Cuvier (1804a: pl. 19) (Fig. 3), est d'ailleurs très comparable à celle publiée en 1789 dans le Supplément de l'Histoire naturelle (Fig. 4A, B) : les deux foramens mentonniers, pourtant très variables chez Procavia, sont positionnés de façon similaire sur le dentaire; à l'arrière du foramen infra-orbitaire on note deux foramens accessoires sur le maxillaire; et les troisièmes molaires sont au même stade de croissance (seule la partie antérieure de la $\mathrm{M}_{3}$ est visible et la $\mathrm{M}^{3}$ est encore dans sa crypte osseuse). De rares disparités sont toutefois observées entre les deux gravures, nous y reviendrons.

Il apparaît ainsi que le crâne de Sidon a occupé une place de choix dans l'échantillonnage de Cuvier. C'est en partie grâce à lui que l'anatomiste a pu réunir, en une seule et même espèce, le daman du Cap et celui du Proche-Orient (le «daman-Israël » que Buffon considérait comme une espèce distincte, quoique proche du daman du Cap). Mais le point essentiel est que, par un examen détaillé du squelette, en particulier crânien, il démontre la proximité du daman avec les Pachydermes (qui comprennent, dans l'acception de l'époque, l'éléphant, le rhinocéros, l'hippopotame, le tapir, et le cochon; Geoffroy SaintHilaire \& Cuvier 1795: 189). Il en fait plus précisément « un intermédiaire entre le rhinocéros et le tapir». Cette position est réaffirmée en 1817, en dépit de quelques modifications apportées à la composition des Pachydermes : Cuvier subdivise alors cet ordre en trois familles, celle des "pachydermes ordinaires" (caractérisée par «quatre, ou trois, ou deux doigts à leurs pieds») étant elle-même scindée en divers sous-groupes, dont celui qui associe le daman aux rhinocéros, au Palaeotherium Cuvier, 1804 (genre fossile) et aux tapirs. Selon Cuvier, ces genres partagent une morphologie similaire des prémolaires et molaires (les "mâchelières»). Il précise à propos des damans, qu' "en les examinant bien, on trouve qu'à la corne près, ce sont en quelque sorte des rhinocéros en miniature» (Cuvier 1817: 240).

Cuvier a donc été le premier à rapprocher les hyracoïdes de certains périssodactyles (au sens moderne du terme). Après lui, la relation hyracoïdes-périssodactyles, voire l'intégration des hyracoïdes au sein de l'ordre des Perissodactyla est longtemps restée une hypothèse soutenue par les zoologistes et paléontologues (Owen 1868: 843), et ce même au cours du XXe siècle (Fischer 1989, Prothero et al. 1988, Thewissen \& Domning 1992). Une autre hypothèse, proposée par Simpson (1945), s'est toutefois imposée aujourd'hui; elle admet une relation entre hyracoïdes, proboscidiens et siréniens au sein du clade Paenungulata. Ce clade est d'ailleurs soutenu par toutes les analyses moléculaires et paléontologiques récentes. Ainsi, bien que les Pachydermes de Cuvier soient clairement un groupe polyphylétique, la relation qu'établissait ce groupe entre hyracoïdes et proboscidiens n'était pas dénuée de sens. À ce propos, on peut remarquer que Cuvier, dans la première édition de la "description ostéologique et comparative du daman", indiquait que les ongles des damans «représentent [...] trèsbien en petit ceux de l'éléphant, tant par leur figure que par la manière dont ils sont placés sur le pied" (Cuvier 1804a: 179). Or, dans la seconde édition de ce travail, telle qu'elle apparaît dans les «recherches sur les ossements fossiles de quadrupèdes ", Cuvier modifie son texte et écrit à propos du daman qu' «il a des ongles arrondis et qui rappellent trèsbien en petit les sabots du rhinocéros". Le mot "éléphant»a donc été remplacé par «rhinocéros». Ce revirement, qui en appellera d'autres pendant près de deux siècles, est en quelque sorte un résumé de toute l'histoire taxinomique supraordinale des hyracoïdes.

\section{LE CRÂNE DÉCRIT PAR DAUBENTON DANS L'HISTOIRE NATURELLE \\ Animal inconnu, loris ou daman?}

Le crâne de Sidon aura donc joué un rôle important dans l'histoire des conceptions sur les damans. Daubenton fut le premier à le décrire, en 1767 , dans le cadre de sa collaboration à l'Histoire naturelle, ouvrage entrepris avec Buffon depuis 1749. Il s'agissait à l'origine d'un projet de catalogue des collections conservées au Cabinet du Jardin du Roi, dont Buffon était intendant depuis 1739 (Roger 1989). Lors de sa nomination, Maurepas l'avait chargé d'entreprendre ce travail, sans doute dans le but de glorifier la richesse des collections et le mécénat royal. Mais à la suite d'un cheminement dont on ignore pratiquement tout, Buffon, sans renoncer à l'idée de catalogue, prit l'initiative d'un projet beaucoup plus ambitieux de description de la nature tout entière, avec des textes généraux proposant des théories nouvelles, portant sur des sujets aussi divers que la reproduction des animaux, la formation de la Terre, la variété des populations humaines, etc. Initialement, il pensait pouvoir tout traiter en quinze volumes 
(voir le prospectus dans Buffon 2007: 843-849), mais il s'avéra rapidement que le travail était beaucoup plus considérable. En définitive, Buffon ne parviendra jamais à terminer son ouvrage, en dépit des trente-six volumes parus de 1749 à 1789 , qui ne couvriront, outre les questions générales, que l'homme, les quadrupèdes vivipares, les oiseaux et les minéraux.

Pour se décharger d'une partie du travail et se réserver ce qu'il jugeait le plus intéressant, Buffon s'adjoignit des collaborateurs, comme Guéneau de Montbeillard et Bexon pour l'Histoire naturelle des oiseaux, et surtout Daubenton, qui joua un rôle particulièrement important dans les quinze premiers volumes, consacrés à l'homme et aux quadrupèdes (Farber 1975; Loveland 2006; Schmitt in Buffon 2009: 10-40 et 2010: 44-71). Dès le début des années 1740, Buffon avait fait venir au Jardin du Roi ce médecin originaire comme lui de Montbard et lui avait confié la gestion des collections, avec le titre de "garde et démonstrateur». C'est donc naturellement à lui qu'il s'adressa pour rédiger les parties purement descriptives de l'Histoire naturelle et ce qui resta du projet initial de catalogue. Ainsi, dès le troisième volume, paru en 1749, Daubenton donna une description très détaillée des objets du Cabinet royal relatifs à l'espèce humaine (Buffon $\&$ Daubenton 1749: 1-304; Buffon 2009: 89-342). À partir du quatrième volume (1753), entamant la série des quadrupèdes, chaque espèce fit l'objet de trois chapitres : une "histoire», par Buffon (traitant des questions générales, des aspects biologiques divers, du comportement, de l'éventuelle utilité, etc.); puis une "description", par Daubenton, présentant d'une manière extrêmement précise la morphologie et l'anatomie de l'animal, avec des tableaux de mesures; et enfin une liste des objets du Cabinet relatifs à l'espèce considérée, numérotés et décrits de manière plus ou moins précise selon les cas, avec parfois des indications sur leur origine. Cette collaboration cessa avec la fin des quadrupèdes, en 1767 , et pour la suite Buffon, qui voyait le nombre de volumes de son ouvrage dépasser considérablement ses prévisions, préféra limiter drastiquement les descriptions anatomiques et se passer des services de Daubenton.

Le travail anatomique et muséologique effectué par ce dernier entre 1749 et 1767 est quantitativement et qualitativement très important, non seulement par la masse énorme de données inédites, mais aussi parce qu'il est sous-tendu par une véritable théorie de la description scientifique (Reynaud 1990). Daubenton souligne en particulier la nécessité d'adopter un style rigoureux et clair, d'employer une nomenclature précise et sans ambiguïté et de suivre un plan toujours identique permettant une comparaison facile entre les espèces. Ces principes seront repris en grande partie par la suite, par Vicq d'Azyr notamment, et joueront un rôle majeur dans l'émergence de l'anatomie comparée (Schmitt 2009, 2016).

La description de la "tête décharnée d'un animal inconnu aux Naturalistes", qui s'avérera être un daman, se trouve dans le dernier volume auquel a contribué Daubenton, paru en 1767 (Buffon \& Daubenton 1767: 205-206). Il s'agit du dernier objet, numéroté «MDCII» (soit 1602), d'un ensemble de "choses arrivées au Cabinet depuis l'impression des Articles auxquels elles ont rapport». La notice descriptive de Dauben-
Daman Hyrax. Tom.3.P2.19
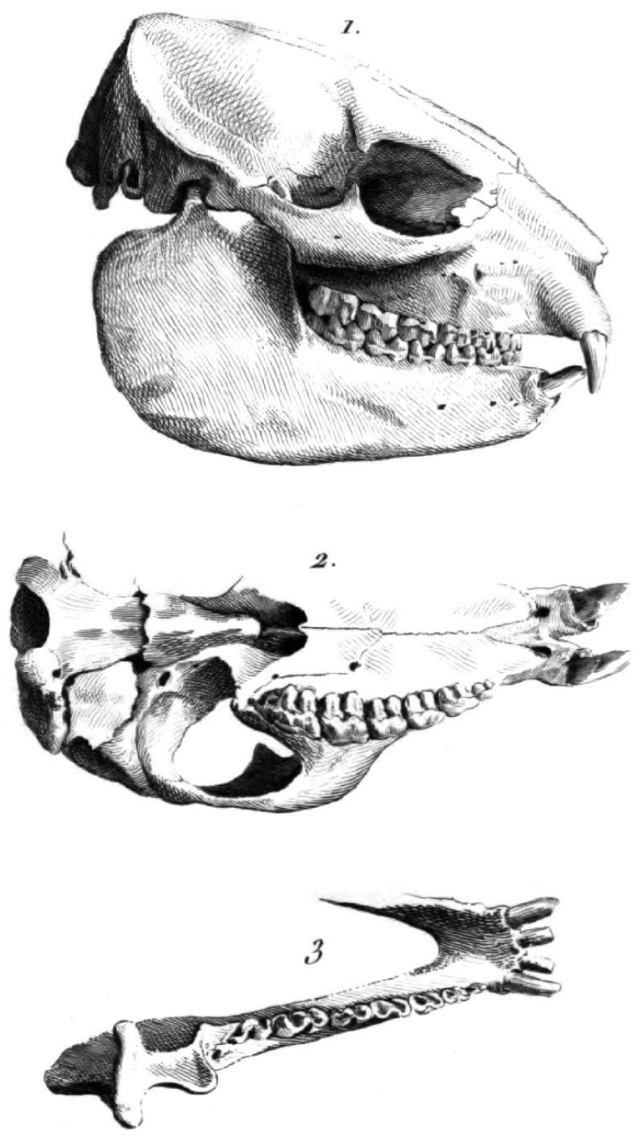

Cimen det

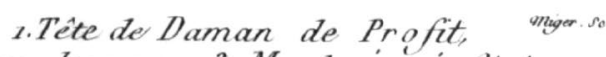

2. oue en dessous; 3. Machoine inférieure.

FIG. 3. - Reproduction de la planche 19 de Cuvier (1804a) figurant le Daman ou Hyrax (= Procavia capensis (Pallas, 1766)), crâne MNHN-ZM-AC-A.2339.

ton est un texte court (37 lignes), rédigé dans un style clair, concis et précis, conformément aux principes généraux qu’il a édictés dès 1749. La description est suivie d'un tableau de mesures, également bref mais semblable, par la forme, le choix et l'ordre des valeurs indiquées, à tous ceux que Daubenton a publiés sur les autres espèces de quadrupèdes. Mais l'une des particularités de cet article est liée au caractère « décharné» du spécimen qui a conduit Daubenton à décrire un mammifère inconnu uniquement à partir de l'étude ostéologique de ses restes crâniens, ce qui constitue une pratique singulière au $\mathrm{XVIII}^{\mathrm{e}}$ siècle; cela deviendra évidemment plus courant dès le début du XIXe siècle avec l'émergence de la paléontologie des vertébrés.

D'emblée Daubenton reconnaît dans le crâne de Sidon un "adulte, car toutes les parties sont bien formées, à l'exception des arrière-dents qui paroissent seulement au bord de leurs alvéoles " : les troisièmes molaires sont en effet en cours de croissance; sur le dentaire, seule la partie antérieure de la $\mathrm{M}_{3}$ (le trigonide) est visible (Fig. 4A). Il extrapole très 
correctement la taille de l'animal grâce «aux dimensions de la tête, comparée à celle des animaux connus; il paroît qu'il étoit de la taille d'un chat ou d'un lièvre" : Procavia capensis mesure en effet entre 39 et $58 \mathrm{~cm}$ et pèse entre 1,8 et $5,5 \mathrm{~kg}$ (Shoshani et al. 2013). La description ostéologique du crâne proprement dite est courte, mais les quelques caractères cités sont pertinents et correspondent de fait à quelques caractères remarquables des hyracoïdes : les frontaux sont larges et plats ("le front est large \& aplati»), le jugal et le pariétal sont proches l'un de l'autre à l'arrière de l'orbite («il n'y a que deux lignes d'intervalle [soit environ $4,5 \mathrm{~mm}$ ] entre les extrémités des apophyses orbitaires des os du front $\&$ de la pomette») et le dentaire, notamment sa partie angulaire, est très développé ( "les branches de la mâchoire du dessous sont très-larges»).

Le reste de la description est consacré à la morphologie des dents et à quelques comparaisons. Daubenton donne tout d'abord la formule dentaire : «les dents incisives sont au nombre de deux à la mâchoire du dessus, $\&$ de quatre à celle du dessous; il n'y a point de dents canines »; il ajoute « la mâchoire supérieure a sept dents mâchelières de chaque côté, \& la mâchoire inférieure seulement six ». Selon la nomenclature actuelle, cette formule dentaire doit se lire : $\mathrm{I} 1 \frac{1}{2}, \mathrm{C} \%, \mathrm{P} 4 / 3$, $\mathrm{M} 3 / 3$. En soi, cette formule peut effectivement correspondre à celle de Procavia capensis, plus exactement aux populations qui ne possèdent pas de $P_{1}$ (e.g., Asher et al. 2017). Toutefois, la gravure publiée dans le Supplément à l'Histoire naturelle révèle au contraire que cette $\mathrm{P}_{1}$ est présente sur le spécimen, au moins sur son hémi-mandibule gauche (Fig. 4A). Ainsi, très étonnamment, il semble que Daubenton ait mal jugé le nombre de dents présentes sur la mandibule. Cela est confirmé par la description de Cuvier (1804a: 177) : la mandibule porte «sept dents d'un côté, et l'alvéole de la première déjà vide de l'autre»; autrement dit, la $\mathrm{P}_{1}$ est présente à gauche (selon la gravure) et la $\mathrm{P}_{1}$ est absente à droite bien que son alvéole soit visible.

Daubenton compare ensuite les prémolaires et molaires du crâne à celles de certains artiodactyles («les mâchelières ont beaucoup de rapport avec celles des animaux ruminans, tels que le bélier, le bouc, les gazelles, \&c»). Cette comparaison implique qu'il avait sans doute remarqué le caractère « ongulé-herbivore» des dents qu'il décrivait. Toutefois, il est admis aujourd'hui que la sélénodontie qui définit les artiodactyles cités par Daubenton est assez différente de la bilophodontie de Procavia. Cette morphologie dentaire, typique des damans, se retrouve par exemple chez le rhinocéros et le tapir. Mais c'est là un argument que Daubenton ne pouvait avancer car les morphologies dentaires de ces deux grands périssodactyles n'étaient pas connues; elles seront publiées en détail plus tard, respectivement par Camper (1780) et Cuvier (1804b). L'essentiel ici est que Daubenton a comparé les molaires de son animal inconnu à certains ongulés; cette idée-force préfigure ainsi les conclusions que Cuvier proposera à propos du daman, près de quarante années plus tard.

Daubenton consacre également plusieurs lignes à la description des incisives, si caractéristiques de Procavia («les incisives sont fort extraordinaires»). Il mentionne par exemple très justement que les inférieures sont proclives («dirigées obliquement en avant et en haut») et légèrement pectinées ("elles ont quatre petits lobes à l'extrémité»). Quant aux incisives supérieures, elles sont reconnues à juste titre comme étant carénées, trièdres («elles ont trois faces longitudinales») et profondément enracinées dans le maxillaire (elles "pénètrent, dans les alvéoles, de la longueur de quinze lignes [soit plus de $3 \mathrm{~cm}$ )»). Signalons enfin que Daubenton a déchaussé les incisives supérieures pour en décrire les racines, encore ouvertes chez l'adulte (« elles sont creuses à la racine»). Cette particularité, qui témoigne d'une croissance continue (hypsodontie) de l'incisive, est un des caractères principaux des hyracoïdes. Ces incisives hypsodontes sont notamment utilisées comme moyen de défense ou d'intimidation (Sale 1966); Daubenton les compare d'ailleurs aux «défenses inférieures des sangliers \& des cochons».

Daubenton précise enfin l'origine du spécimen : cette tête, écrit-il, a été "trouvée dans un puits desséché de l'ancienne Sidon, et envoyée à feu M. le Comte de Caylus, qui l'a donnée au Cabinet». Le donateur était donc Anne-Claude Philippe de Tubières-Grimoard de Pestel de Levis, comte de Caylus (1692-1765), soldat, voyageur, artiste et collectionneur français, passionné d'archéologie, qui avait voyagé en Orient dans sa jeunesse. On ignore si l'objet avait été acquis par Caylus à l'occasion de ce séjour, ou s'il le reçut en France par la suite. La date de sa remise au Cabinet du Roi est également inconnue : on sait seulement qu'elle est survenue avant la mort du comte de Caylus, le 5 septembre 1765, puisque Daubenton parle expressément d'un don de la part de l'aristocrate.

En revanche, il est clair que le texte de Daubenton, lui, fut rédigé (ou du moins achevé) après la mort de Caylus, puisqu'il est précisé « feu M. le Comte de Caylus ». Le quinzième volume de l'Histoire naturelle étant paru au plus tard en avril 1767 (Mercure de France, avril 1767: 120), compte tenu des délais de composition et d'impression de l'ouvrage, celui-ci a sans doute été remis à l'éditeur fin 1766. Daubenton n'a donc manifestement guère eu le temps de consulter la première description précise d'un daman publiée par Pallas dans les Miscellanea zoologica, dont la dédicace est datée du 18 octobre 1766. S'il en avait eu l'occasion, on peut imaginer qu'il aurait pu faire le rapprochement entre les deux exemplaires, d'autant que la planche de Pallas montre clairement la tête de face et les incisives si particulières de l'animal. Mais il se serait sans doute heurté à une difficulté, celle du nombre de «molaires " (prémolaires et molaires) : le spécimen décrit par Pallas était en effet un très jeune individu mais non reconnu comme tel, ayant quatre prémolaires de lait molariformes (voir plus haut), alors que celui de Daubenton était un adulte ayant toute sa denture adulte visible. Pallas, de son côté, ne pouvait évidemment pas avoir lu la notice de Daubenton lorsqu'il publia cette première version de sa description du "Cavia capensis», et il est même douteux qu'il ait pu en prendre connaissance avant de rendre le manuscrit de la seconde, dans le second fascicule des Spicilegia zoologica de 1767. Les deux savants ont donc travaillé de manière totalement indépendante, et il faudra attendre près de dix ans pour que, dans une courte note sans équivoque, additionnelle à son article de 1767, Pallas (1776: 85-86) reconnaisse un daman dans le crâne de Sidon décrit par Daubenton. 

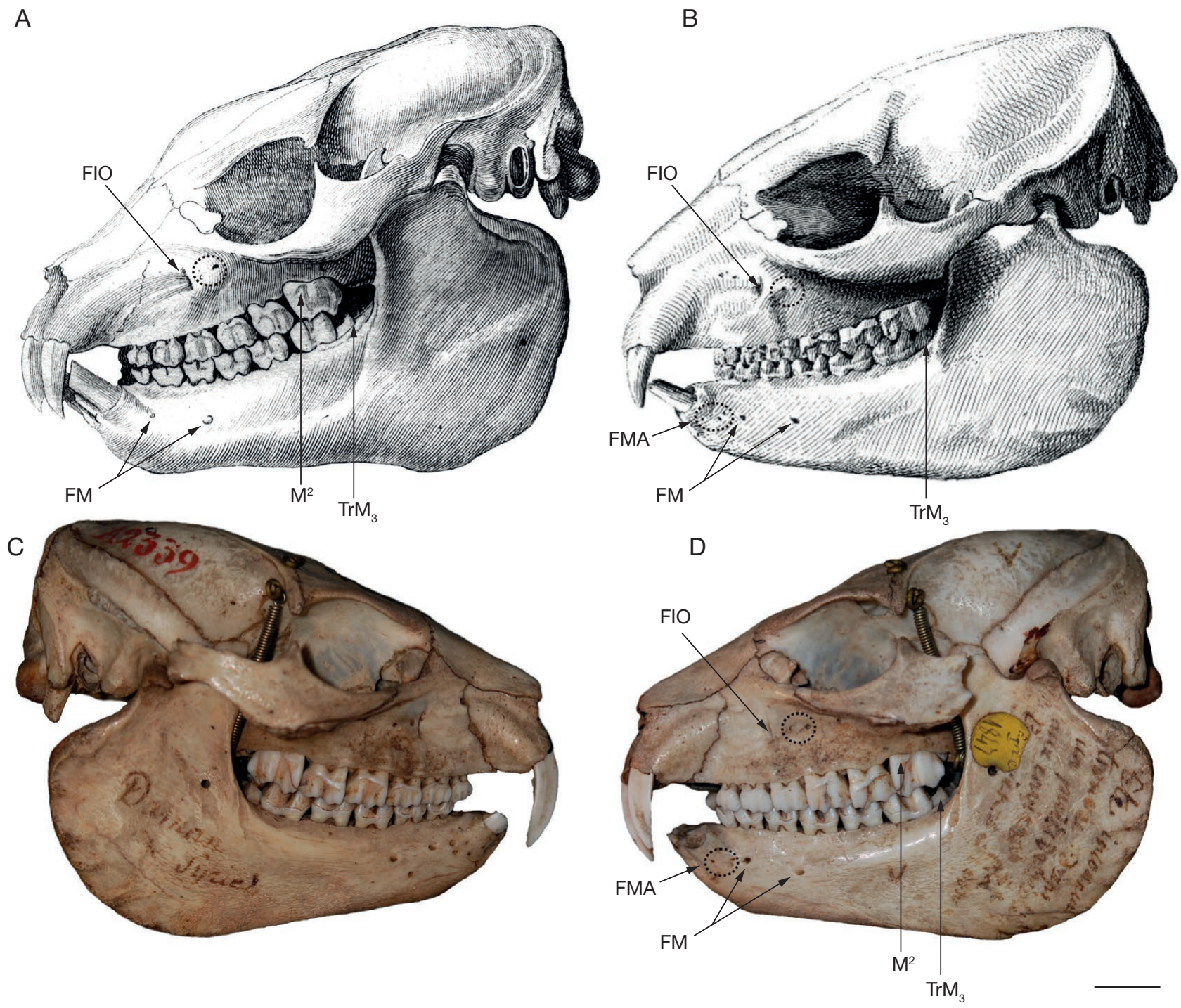

FIG. 4. - Crâne de Procavia capensis (Pallas, 1766) MNHN-ZM-AC-A.2339 : A, d'après la planche XXXVII du Supplément de l'Histoire naturelle (tome 7, publié en 1789) (Buffon 1789); B, d'après la planche 19 de Cuvier (1804a); C, D, photos du spécimen (C, vue latérale droite et $\mathbf{D}$, vue latérale gauche). A et $\mathbf{B}$ sont inversées pour faciliter la comparaison avec $\mathbf{D}$; les similitudes morphologiques observées sont: le degré de croissance des dernières molaires (seul le trigonide de la $\mathrm{M}_{3}$ est visible et la $\mathrm{M}^{3}$ est dans sa crypte osseuse), l'échancrure du maxillaire au-dessus de la $\mathrm{M}^{2}$ (ce caractère est assez unique au niveau spécifique), et la position des deux foramens mentonniers principaux (sous la racine antérieure de la $\mathrm{P}_{2}$ et sous $\mathrm{P}_{3}-\mathrm{P}_{4}$ ), celle des trois foramens mentonniers accessoires (sous et à l'avant de la $\mathrm{P}_{1}$ ) et celle des deux petits foramens à l'arrière du foramen infra-orbitaire sur le maxillaire. Abréviations : FIO, foramen infraorbitaire; FM, foramens mentonniers; FMA, foramens mentonniers accessoires; $\mathbf{M}^{2}$, seconde molaire supérieure; $\mathbf{T r M}_{3}$, trigonide de la troisième molaire inférieure. Échelle : $1 \mathrm{~cm}$.

En revanche, Buffon, dans ses nouveaux articles sur le daman de 1776 et surtout de 1782 , ne fait aucune référence au crâne. Ce dernier, pourtant, va réapparaitre en 1789 dans le dernier volume du Supplément à l'Histoire naturelle, édité après la mort de Buffon par Lacépède : non pas dans l'article consacré alors au daman ou "marmotte du Cap" (qui, comme nous l'avons vu plus haut, marque en fait un recul par rapport à celui de 1782), mais en étant attribué à un tout autre animal, le "loris du Bengale», c'est-à-dire Nycticebus bengalensis, qui est un primate. Cette fois, le crâne est représenté sur une planche dessinée par Jacques De Sève (le principal illustrateur de l'Histoire naturelle) et gravée par le fils de ce dernier, avec une dent venue de Chine, envoyée en 1771 par le naturaliste allemand Peter Hinrich Tesdorff et attribuée au même animal (Buffon 1789: 134-135 et pl. XXXVII). Outre la lettre de Tesdorff, cet article s'appuie principalement sur une monographie de Vosmaer (1770), qui a considéré le loris en question comme un "paresseux", une interprétation contestée par Buffon, qui cite cependant longuement la description du naturaliste néerlandais.

Nulle justification n'est donnée à l'attribution du crâne de Sidon à ce loris, qui est en vérité assez curieuse, car malgré quelques similitudes, la morphologie, notamment dentaire, des deux animaux diverge nettement. Ainsi, le "loris de Bengale» se caractérise par une mandibule portant " quatre dents incisives étroites et plates, suivies des deux côtés d'une plus grande et 

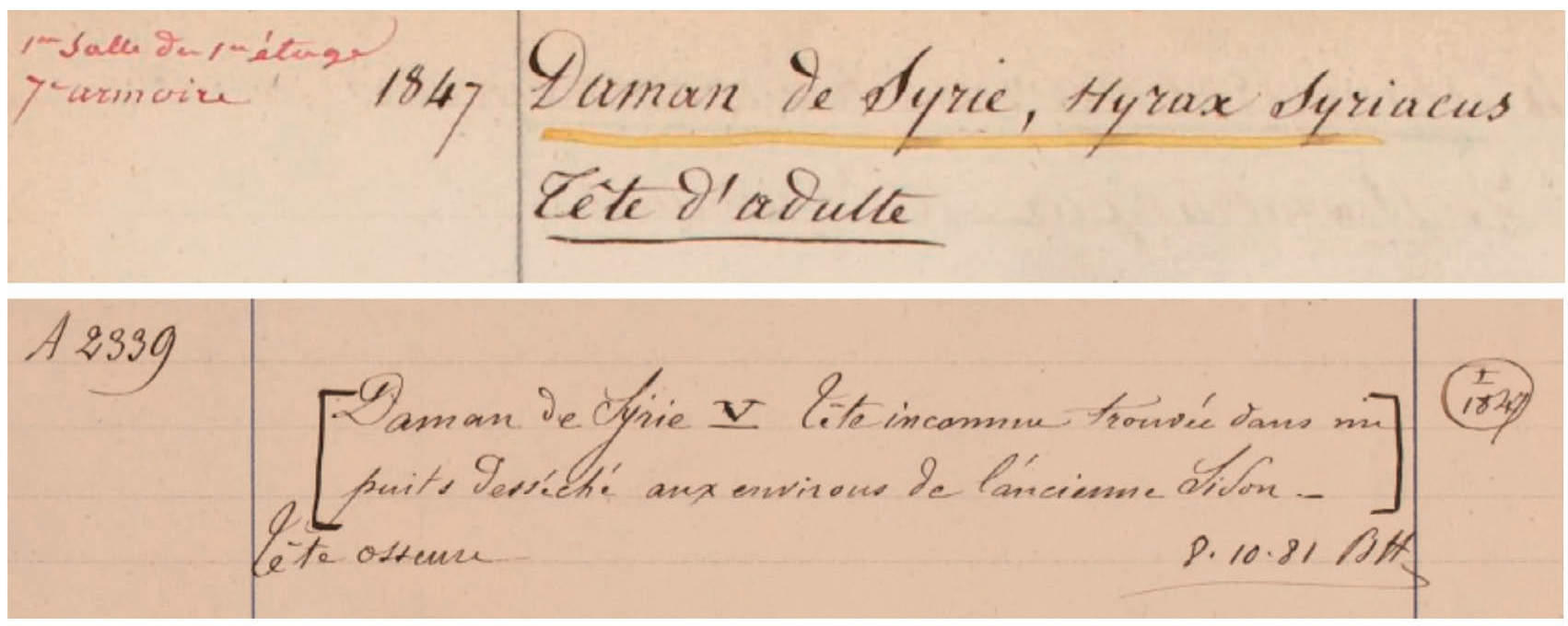

FIG. 5. - Extraits des catalogues du MNHN faisant mention du crâne de Procavia capensis (Pallas, 1766) MNHN-ZM-AC-A.2339: A, catalogue des préparations anatomiques du Cabinet d'Anatomie comparée du Muséum d'Histoire naturelle, B, catalogue d'Anatomie comparée.

enfin deux grosses dents canines " et un maxillaire portant de chaque côté «deux petites dents écartées» suivies d'une petite canine; le daman, lui, ne porte pas de canine. Cuvier verra d'ailleurs dans cette identification de la tête de Sidon comme un loris "une erreur presque inconcevable», ce crâne n'ayant avec le «loris paresseux du Bengale, [...] aucun rapport ni de grandeur, ni de forme, de composition» (1804a: 172-173).

Il est possible que ce texte, dans lequel n'apparaît aucun élément postérieur à 1775, ait été rédigé, tout comme la notice sur la "marmotte du Cap", de nombreuses années avant leur publication en 1789, car on sait que Lacépède a composé ce volume posthume de manière assez désordonnée, en rassemblant des écrits de Buffon (voire de Daubenton) plus ou moins anciens et pas tous destinés à être publiés (voir Loveland 2006: 483). On ne peut exclure non plus que Buffon ait été influencé par la lettre de Bruce qu'il cite en 1782 à propos du «daman-Israël » et selon laquelle cet animal, par certains caractères (l'absence de queue et la forme des doigts), "paroît approcher du loris» (Buffon 1782: 276); mais il semble étonnant alors qu'il n'ait pas évoqué le daman dans la notice sur le loris publiée en 1789. Peut-être Lacépède a-t-il publié une version inachevée ou incomplète de l'article. Il est en tout cas probable que Daubenton n'ait pris aucune part à cette attribution tardive du crâne de Sidon au loris dans ce Supplément de l'Histoire naturelle de 1789, treize ans après l'identification correcte de Pallas, et s'il en a pris connaissance, il en a vraisemblablement été très surpris. Mais il est vrai qu'à cette date, il ne s'occupait plus guère d'anatomie (Loveland 2004).

\section{Histoire du spécimen depuis 1789 et sa redécouverte}

Pendant la Révolution, alors que Daubenton œuvre à la survie du Jardin du Roi et assure sa transformation en Muséum national d'Histoire naturelle, le crâne de Sidon rejoint tout naturellement les collections de la nouvelle institution. Quelques années plus tard, nous l'avons souligné, il sera l'une des pièces maîtresses de Cuvier (1804a, 1812) dans son argumentaire sur la classification des damans au sein des Pachydermes. Les dernières mentions précises du spécimen sont celles de Blainville (1863: 14-15) et George (1874: 10). Étonnamment, il ne sera jamais cité dans les monographies de Thomas (1892) et Hahn (1934) pourtant destinées à faire le point sur la systématique confuse des hyracoïdes. Seule la synthèse de Fischer (1992), beaucoup plus récente, illustre le spécimen, mais sans plus de précision, en reprenant la planche de 1789.

Dans sa monographie du genre "Daman», George (1874) précise que le crâne est, à cette époque, au Cabinet d'Anatomie comparée du MNHN «sous le $\mathrm{n}^{\circ} \mathrm{I}, 1847$ et porte, inscrite à l'encre, la mention de son origine telle qu'elle y fut mise par Daubenton». Ce numéro correspond à une entrée du Catalogue des préparations anatomiques du Cabinet d'Anatomie comparée; le crâne y était conservé dans la 1 re salle du 1 er étage (Fig. 5A). Selon le Catalogue actuel d'Anatomie comparée, le spécimen «I, 1847 » possède aujourd'hui le numéro MNHNZM-AC-A.2339 (Fig. 5A); il est exposé dans la Galerie de Paléontologie et d'Anatomie comparée. Depuis l'ouverture de celle-ci en 1898, le spécimen est présenté dans la vitrine des "pachydermes ", quelques mètres à droite du buste de Cuvier, avec d'autres restes crâniens et postcrâniens d'hyracoïdes. Sans commentaire particulier sur le rôle capital qu'il a joué dans l'histoire des conceptions sur les hyracoïdes, il porte le nom de "Hyrax syriacus" (aujourd'hui Procavia capensis syriaca). Son entrée dans le Catalogue des Collections d'Anatomie comparée, datée de 1881, ne fait pas non plus référence à son historique (Fig. 5B).

Comme signalé par George (1874), le crâne porte inscrit sur l'hémi-mandibule gauche l'annotation "tête inconnue trouvée dans un puits desséché aux environs de l'ancienne Sidon "; cette annotation évoque incontestablement l'article de Daubenton publié dans l'Histoire naturelle (Fig. 6). Outre cet argument, la certitude que le crâne MNHN-ZM-AC- 
A.2339 est bien celui décrit par Daubenton se fonde sur ses mesures (très proches de celles publiées dans l'Histoire naturelle) et sur l'ensemble des caractères, déjà cités plus haut, qui sont visibles sur la gravure publiée dans les Suppléments de l'Histoire naturelle (degré de croissance des dernières molaires et nombre et position des foramens mentonniers sur le dentaire et des foramens accessoires à l'arrière du foramen infra-orbitaire) (Fig. 4). À cela s'ajoute la forte échancrure du maxillaire au-dessus de la $\mathrm{M}^{2}$; ce caractère est assez unique au niveau spécifique. On retrouve également sur MNHN-ZMAC-A.2339 les trois petits foramens mentonniers accessoires sous et à l'avant de la $\mathrm{P}_{1}$ comme illustrés par Cuvier. À ce titre, MNHN-ZM-AC-A.2339 présente, comme constaté par Cuvier (contra Daubenton), une $\mathrm{P}_{1}$ gauche et l'alvéole de celle-ci à droite (Fig. 4C, D). Notons également que le contour du dentaire, du côté ventral, est plus conforme à la gravure donnée par Cuvier qu'à celle publiée dans les Suppléments de l'Histoire naturelle. Inversement, la gravure des Suppléments est plus correcte en ce qui concerne la partie rostrale du crâne (longueur et contour des incisives supérieures et présence de la suture prémaxillaire-maxillaire). Depuis sa première description, le crâne est resté dans un état de conservation assez remarquable; seules les incisives inférieures sont cassées ( $\mathrm{I}_{1}$ gauche et $\mathrm{I}_{2}$ droite) ou manquantes ( $\mathrm{I}_{1}$ droite et $\mathrm{I}_{2}$ gauche) et la région de la suture jugal-squamosal, des côtés droit et gauche, est brisée (Fig. 4). Ajoutons enfin que le numéro d'inventaire d'origine (MDCII), tel qu'il est mentionné dans l'Histoire naturelle, n'est pas visible sur le spécimen (a-t-il été effacé ou n'a-t-il jamais été inscrit? Rien ne permet de répondre à ces interrogations).

\section{CONCLUSION}

L'identification du spécimen MNHN-ZM-AC-A.2339 revêt donc un double intérêt. D'une part, il subsiste peu d'objets de l'ancien Cabinet royal, du moins qui soient actuellement identifiés comme tels, et la «redécouverte » de l'un d'eux constitue un événement notable. D'autre part, ce crâne joua un rôle significatif dans l'histoire de la mammalogie : il s'agit en effet du premier crâne d'hyracoïde publié dans la littérature et, si Daubenton ne fut pas en mesure d'identifier l'animal dans sa notice parue en 1767 dans l'Histoire naturelle, il distingua d'emblée en lui plusieurs caractères remarquables du groupe. Par la suite, ce même crâne fut reconnu par Pallas, en 1776, puis illustré (mais attribué à un autre animal) en 1789 dans le Supplément de l'Histoire naturelle. Enfin, Cuvier, qui l'étudia de nouveau en 1804, s'en servit, avec d'autres spécimens, pour démontrer la proximité anatomique du daman et des "pachydermes", ouvrant la voie à de réelles avancées dans la systématique des mammifères.

Ainsi, en quatre décennies à peine, cet objet, offert au Cabinet du Roi par un aristocrate collectionneur qui n'imaginait sans doute pas son importance scientifique, aura contribué aux progrès considérables de l'anatomie comparée, entre les mains de deux des principaux acteurs de ces progrès, Daubenton et Cuvier.

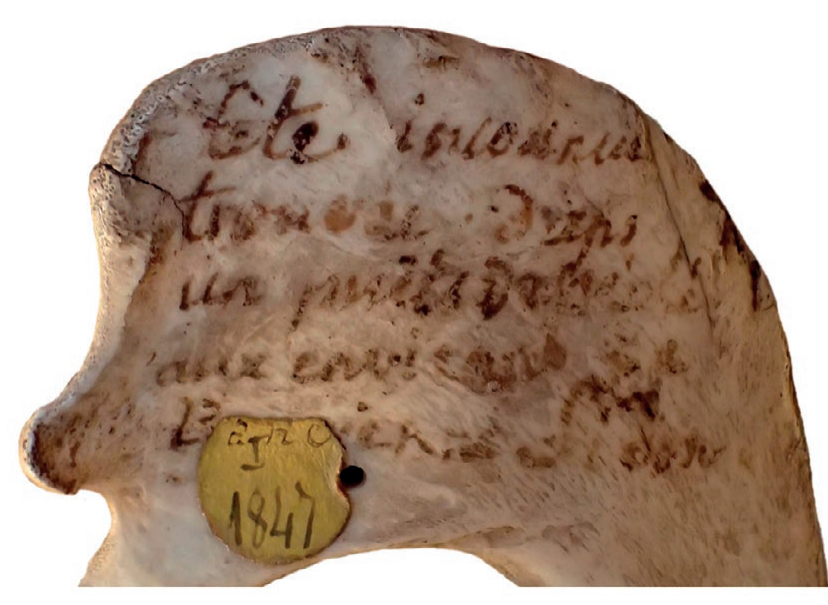

FIG. 6. - Détail du crâne de Procavia capensis (Pallas, 1766) MNHN-ZM-ACA.2339 montrant l'inscription "tête inconnue trouvée dans un puits desséché aux environs de l'ancienne Sidon ". Outre les arguments morphologiques (voir Fig. 4 et partie «Résultats et Discussion » pour plus de détails), cette inscription témoigne que le spécimen MNHN-ZM-AC-A.2339 est bien le crâne décrit par Daubenton dans son article de 1767 intitulé "la tête décharnée d'un animal inconnu aux Naturalistes » découvert dans un puits asséché de la ville antique de Sidon (Buffon \& Daubenton 1767: 205-207).

\section{Remerciements}

Nous remercions vivement Joséphine Lesur, Luc Vives, Christine Argot, Céline Bens et Guillaume Billet (MNHN) pour leur accueil dans les collections et leur aide précieuse durant la rédaction de cet article. Nous remercions également Kees Rookmaaker (Rhino Research Center) pour l'envoi de documentation bibliographique et Myriam Boivin (Université de Montpellier) pour le prêt de spécimens de rongeurs cavioïdes. Nous remercions enfin les deux rapporteurs de cet article, dont Pascal Tassy (MNHN), pour leur relecture précieuse et leurs suggestions.

\section{RÉFÉRENCES}

AlPINI P. 1735. - Historia Agypti naturalis pars prima. Qua continentur Rerum Egyptiarum libri quatuor. Opus posthumum, nunc primum. Gérard Potuliet, Leyde, 260 p. http://gdz.sub. uni-goettingen.de/dms/load/toc/?PID=PPN490536654

ASHER R. \& SEIFFERT E. R. 2010. — Systematics of Endemic African Mammals, in SANDERS W. J. \& Werdelin L. (eds), Cenozoic Mammals of Africa. University of California Press, Berkeley: 903-920.

Asher R., Gunnell G., Seiffert E. R., Pattinson D., Tabuce R., Hautier L. \& Sallam H. M. 2017. - Dental eruption and growth in Hyracoidea (Mammalia, Afrotheria). Journal of Vertebrate Paleontology e1317638. https://doi.org/10.1080/02724 634.2017.1317638

BALAN B. 1979. - L'ordre et le temps. L'anatomie comparée et l'histoire des vivants au XIXe siècle. Vrin, Paris, $610 \mathrm{p}$.

Beaulieu A. DE 1664. - Mémoires du voyage aux Indes Orientales, in THÉVENOT M. (ed.), Relations de divers voyages curieux, qui n'ont point esté publiees; ou qui ont esté traduites. Volume 2. Cramoisy, 128 p. http://gallica.bnf.fr/ark:/12148/bpt6k9627352r

BlainVIlle H. M. DuCROTAY DE 1863. — Ostéographie ou description iconographique comparée du squelette et du système dentaire des mammiferes récents et fossiles pour servir de base à la zoologie et à la géologie. Tome 3: Quaternatés: des damans (Buffon). Baillière, Paris, 850 p. http://gallica.bnf.fr/ark:/12148/bpt6k62228330?rk=128756;0 
BRUCE J. 1790. - Travels to Discover the Source of the Nile: In the Years 1768, 1769, 1770, 1771, 1772, and 1773, vol. 1. Ruthven, Édimbourg, 535 p. https://doi.org/10.5962/bhl.title.26045

BUFFON G.-L. LECLERC COMTE DE. 1776. - Histoire naturelle, générale et particulière. Servant de suite à l'histoire des Animaux quadrupèdes. Supplément. Tome troisième. Imprimerie Royale, Paris, $255 \mathrm{p}$.

BufFon G.-L. LeClerC COMTE DE. 1778. - Histoire naturelle, générale et particulière [...]. Supplément. Tome quatrième. Nouvelle édition. Schneider, Amsterdam, 174 p.

BuFFON G.-L. LECLERC COMTE DE. 1782. - Histoire naturelle, générale et particulière. Supplément. Tome sixième. Imprimerie Royale, Paris, 430 p.

BufFON G.-L. LEClERC COMTE DE. 1789. - Histoire naturelle, générale et particulière, servant de suite à l'histoire des Animaux quadrupèdes. Supplément. Tome septième. Imprimerie Royale, Paris, $364 \mathrm{p}$.

BufFon G.-L. LeClerC COMTE DE. 2007. - Euvres complètes. Vol. 1. Édité par Schmitt S. et Crémière C. Honoré Champion, Paris, 10 vol. parus, $1376 \mathrm{p}$

BufFON G.-L. LeCLERC COMTE DE. 2009. - Euvres complètes. Vol. 3. Édité par Schmitt S. et Crémière C. Honoré Champion, Paris, $768 \mathrm{p}$

Buffon G.-L. LeClerC COMTE DE. 2010. - Euvres complètes. Vol. 4. Édité par Schmitt S. et Crémière C. Honoré Champion, Paris, $862 \mathrm{p}$.

BufFon G.-L. LeClerC COMTE DE. 2016. - Euvres complètes. Vol. 9. Édité par Schmitt S. et Crémière C. Honoré Champion, Paris, $719 \mathrm{p}$.

BufFON G.-L. LeCLERC COMTE DE \& DAUBENTON L.-J.-M. 1749. Histoire naturelle, générale et particulière, avec la description $d u$ Cabinet du Roi. Tome troisième. Imprimerie Royale, Paris, 530 p.

BufFON G.-L. LeCleRC COMTE DE \& DAUBENTON L.-J.-M. 1765. Histoire naturelle, générale et particulière, avec la description $d u$ Cabinet du Roi. Tome treizième. Imprimerie Royale, Paris, 441 p.

BuFFON G.-L. LECLERC COMTE DE \& DAUBENTON L.-J.-M. 1767. Histoire naturelle, générale et particulière, avec la description $d u$ Cabinet du Roi. Tome quinzieme. Imprimerie Royale, Paris, $530 \mathrm{p}$.

Callou C., Cuisin J. \& Groves C. 2010. - The rediscovery of Buffon's tarsier. International Journal of Primatology, 31: 10551070 https://doi.org/10.1007/s10764-010-9455-x

CAMPER P. 1780. - Dissertatio de cranio Rhinocerotis a fricani, cornu gemino; Academiae Scientiarum Imperiali Petropolitanae oblata. Acta Academiae Scientiarum Imperialis Petropolitanae 1 (2): 193-209.

Cuvier G. 1800. - Leçons d'anatomie comparée. Tome II. Contenant les organes des sensations. Baudouin, Paris, 698 p. http://gallica. bnf.fr/ark:/12148/bpt6k64789154

CUVIER G. 1804a. - Description ostéologique et comparative du Daman Hyrax Capensis. Annales du Muséum national d'Histoire naturelle 3: 171-182.

Cuvier G. 1804b. - Description ostéologique du Tapir. Annales du Muséum national d'Histoire naturelle 3: 122-131.

CUVIER G. 1812. — Recherches sur les ossemens fossiles de quadrupèdes. Tome second. Déterville, Paris. http://gallica.bnf.fr/ark:/12148/ bpt6k10577464

CUVIER G. 1817. - Le règne animal distribué d'après son organisation. Tome I. Déterville, Paris, 540 p. https://doi.org/10.5962/ bhl.title. 41460

CUVIER G. 1822. - Recherches sur les ossemens fossiles, où l'on rétablit les caractères de plusieurs animaux, dont les révolutions du globe ont détruit les espèces. Dufour et d'Ocagne, Paris.

Daubenton L.-J.-M. 1782. - Encyclopédie méthodique. Histoire naturelle des animaux. Tome premier. Panckoucke, Paris, xcii-691 p.

FARBER P. L. 1975. - Buffon and Daubenton: Divergent Traditions within the Histoire naturelle. Isis 66: 63-74. https://doi. org/10.1086/351376

ERXLEBEN J. C. P. 1777. — Systema regni animalis per classes, ordines, genera, species, varietates cum synonymia et historia animalium.
Classis I. Mammalia. Weygand, Leipzig, 636 p. https://www. biodiversitylibrary.org/item/53898\#page/7/mode/1up

Fischer von Waldheim G. 1803. - Das Nationalmuseum der Naturgeschichte zu Paris. Von seinem ersten Ursprunge bis zu seinem jetzigen Glanze. Vol. 2. F. Esslinger, Francfort, 422 p. https://doi. org/10.5962/bhl.title.101282

FISCHER M. S. 1989. - Hyracoids, the sister-group of the perissodactyls, in Prothero D. R. \& Schoch R. M. (eds), The Evolution of Perissodactyls. Oxford University Press, New York: 37-56.

FISCHER M. S. 1992. - Hyracoidea. Handbuch der Zoologie, Band VIII Mammalia. Walter de Gruyter, Berlin et New York, 176 p.

Geoffroy SainT-Hilaire É. 1803. - Catalogue des Mammiferes du Muséum national d'histoire naturelle. s.n., s.l. 272 p.

Geoffroy Saint-Hilaire É. \& Cuvier G. 1795. — Mémoire sur une nouvelle division des Mammiferes, et sur les principes qui doivent servir de base dans cette sorte de travail, lu à la société d'Histoire naturelle, le premier floréal de l'an troisième, par les citoyens Geoffroy et Cuvier. Magasin Encyclopédique 1re année, 2: 164-190. https://www.biodiversitylibrary.org/item/30802\#page/9/mode/1up

GEORGE H. 1874. - Monographie anatomique et zoologique des mammiferes du genre Daman. Annales des Sciences naturelles, Zoologie et Paléontologie 1 (9): 1-260. http://gallica.bnf.fr/ark:/12148/ bpt6k96111570

HAHN H. vON 1934. — Die familie der Procaviidae. Zeitschrift für Säugetierkunde 9: 207-358.

HERMANN J. 1783. - Tabula affinitatum animalium, olim academico specimine edita, nunc uberiore commentario illustrata, cum annotationibus ad historiam naturalem animalium augendam facientibus. J. G. Treuttel, Strasbourg, 371 p. https://doi.org/10.5962/bhl. title. 58872

Hondius J. 1652. - Klare Besgryving van Cabo de Bona Esperanca. Hondius, Amsterdam, 33 p.

KLEIN J. T. 1751. - Quadrupedum dispositio brevisque historia naturalis. Jonas Schmidt, Leipzig, 1751, 128 p. https://doi. org/10.5962/bhl.title.49492

KolB P. 1719. - Caput Bone Hodiernum Das ist : Vollständige Beschreibung des africanischen Vorgebürges der Guten Hofnung. Peter Conrad Monath, Nuremberg, 846 p. https://archive.org/ details/bub_gb_MbxYAAAAcAAJ

LiNNAEUS C. VON 1788. - Systema Nature, per Regna tria Nature, secundum Classes, Ordines, Genera, Species, cum Characteribus, Differentiis, Synonymis, Locis. Tomus 1. Editio decima tertia, aucta, reformata. Cura Jo. Frid. Gmelin. G. E. Beer, Leipzig, 500 p. https://doi.org/10.5962/bhl.title.36932

LOVELAND J. 2004. - Daubenton's Lions : From Buffon's Shadow to the French Revolution. New Perspectives on the Eighteenth Century 1: 29-47. https://ahrf.revues.org/13287?lang=en

Loveland J. 2006. - Another Daubenton, Another Histoire naturelle. Journal of the History of Biology 39: 457-491. https:// doi.org/10.1007/s10739-005-6533-6

MeLLIN A. W. VON 1782. - Der Klipdas. Schriften der Berlinischen Gesellschaft naturforschender Freunde 3: 271-284. http://ds.ub. uni-bielefeld.de/viewer/image/1923584_003/289/LOG_0028/

Owen R. 1868. — On the Anatomy of Vertebrates. Vol. III. Mammals. Longmans, Green and Co., x-915 p. https://doi.org/10.5962/ bhl.title.990

Pallas P. S. 1766. - Miscellanea zoologica quibus nove imprimis atque obscure animalium species describuntur. Van Cleef, La Haye, xii-224 p. https://doi.org/10.5962/bhl.title.69851

PALlas P. S. 1767. - Spicilegia zoologica quibus nove et obscure animalium species iconibus, descriptionibus atque commentariis illustrantur. Fasciculus secundus. Lange, Berlin, 32 p. http://gdz. sub.uni-goettingen.de/dms/load/img/?PID=PPN391052802

PALLAS P. S. 1776. - Spicilegia zoologica quibus nove imprimis et obscure animalium species iconibus, descriptionibus atque commentariis illustrantur. Fasciculus undecimus. Voss, Berlin, 86 p. http://gdz.sub.uni-goettingen.de/dms/load/img/?PID=PPN59 5184561\%7CLOG_0005 
Patton J. L., Pardiñas U. F. J. \& D’Elía G. 2015. - Mammal of South America. Volume 2: Rodents. The University of Chicago Press, xxvi + $1336 \mathrm{p}$.

Prothero D. R., Manning E. M. \& Fischer M. S. 1988. The phylogeny of the ungulates, in BENTON M. J. (ed.), The Phylogeny and Classification of the Tetrapods. Clarendon Press, Oxford: 201-234.

REYNAUD D. 1990. - Pour une théorie de la description au $18 \mathrm{e}$ siècle. Dix-huitième Siècle 22: 347-366. https://doi.org/10.3406/ dhs. 1990.1768

Roger J. 1989. - Buffon. Un philosophe au Jardin du Roi. Fayard, Paris, $645 \mathrm{p}$

ROOKMAAKER L. C. 1989. - The Zoological Exploration of Southern Africa 1650-1790. Balkema, Rotterdam, xii-368 p.

SALE J. B. 1966. - Daily food consumption and mode of ingestion in the hyrax. Journal of The East Africa Natural History Society and National Museum 25: 215-224

SCHмiтT S. 2006. - Aux origines de la biologie moderne. L'anatomie comparée d'Aristote à la théorie de l'évolution. Belin, Paris, $464 \mathrm{p}$.

SCHMiTT S. 2009. - From Physiology to Classification: Comparative Anatomy and Vicq d'Azyr's Plan of Reform for Life Sciences and Medicine (1774-1794). Science in Context 22 (2): 145-193. https://doi.org/10.1017/S0269889709002191

SchmitT S. 2016. - Studies on Animals and the Rise of Comparative Anatomy at and around the Parisian Royal Academy of Sciences in the Eighteenth Century. Science in Context 29 (1): 11-54. https://doi.org/10.1017/S026988971500037X

SCHMITT S. À PARAÎTRE. - La nomenclature des animaux dans l'Histoire naturelle, in BufFON G. L. LECLERC COMTE DE, Euvres complètes. Vol. 12. Édité par Schmitt S. et Crémière C., Honoré Champion, Paris: 17-176.

SiMPSON G. G. 1945. - The principles of classification and a classification of mammals. Bulletin of the American Museum of Natural History 85: 1-350.

SHAW T. 1738. - Travels, or Observations relating to several parts of Barbary and the Levant. Theatre, Oxford, xvi-442-60 p. https:// doi.org/10.5962/bhl.title.26044

SHaW T. 1743. - Voyages de Monsr. Shaw, M. D. dans plusieurs provinces de la Barbarie et du Levant. Tome second. J. Neaulme, La Haye, 361 p. http://gallica.bnf.fr/ark:/12148/bpt6k104727s
Shoshani J., Bloomer P., Seiffert E. R. 2013. — Family Procaviidae, in Kingdon J., Happold D., Hoffmann M., Butynski T., Happold M. \& Kalina J. (eds), Mammals of Africa, Volume I: Introductory Chapters and Afrotheria. Bloomsbury Publishing, London: 149-152.

Stanhope M. J., Waddell V. G., Madsen O., Jong W. W. de, Hedge S. B., Clevens G. C., KaO D. \& Springer M. S. 1998. Molecular evidence for multiple origins of Insectivora and for a new order of endemic African insectivore mammals. Proceedings of the National Academy of Sciences USA 95 (17): 9967-9972. https://doi.org/10.1073/pnas.95.17.9967

STORR G. C. C. 1780. — Prodromus methodi animalium. Reiss, Tubingen, $43 \mathrm{p}$.

Tabuce R., Lehmann T. \& Asher R. 2008. - Afrotherian mammals: a review of current data. Mammalia 72: 2-14. https://doi. org/10.1515/MAMM.2008.004

TASSY P. 2002. — L'émergence du concept d'espèce fossile : le mastodonte américain (Proboscidea, Mammalia) entre clarté et confusion. Geodiversitas 24 (2): 263-294.

Thewissen J. G. M. \& Domning D. P. 1992. - The role of phenacodontids in the origin of the modern orders of ungulate mammals. Journal of Vertebrate Paleontology 12 (4): 494-504. https://doi.org/10.1080/02724634.1992.10011476

Thomas O. 1892. - On the species of Hyracoidea. Proceedings of the Zoological Society of London, 1892: 50-76.

VICQ D’AZYR F. 1792. - Encyclopédie méthodique. Systême anatomique. Quadrupèdes. Tome second. Panckoucke, Paris, clxiv-632 p.

VOSMAER A. 1767a. - Beschryving van eene zeer vreemde en geheel nieuwe soort van Africaansch Basterd-Mormeldier. P. Meijer, Amsterdam, $8 \mathrm{p}$.

VOSMAer A. $1767 \mathrm{~b}$. - Description d'une très-étrange et tout-à-fait nouvelle Espèce de Marmote-Bâtarde d'Afrique. P. Meijer, Amsterdam, 8 p. https://doi.org/10.5962/bhl.title.120457

VOSMAer A. 1770. - Description d'une espèce de Paresseux pentadactyle, jusqu'ici inconnu, qui se trouve au Bengale. P. Meijer, Amsterdam, 19 p. https://doi.org/10.5962/bhl.title.120510

Wendland F. 1992. - Peter Simon Pallas, 1741-1811. Materialien einer Biographie, vol. 1. W. de Gruyter, Berlin.

WiedemanN C. R. W. 1802. — Beschreibung des Schädels vom Daman (Hyrax capensis). Archiv für Zoologie und Zootomie 3: 42-51. 
